\begin{tabular}{|c|c|c|c|}
\hline Eiszeitalter u. Gegenwart & 42 & $\begin{array}{c}115-120 \\
7 \mathrm{Abb} .\end{array}$ & Hannover 1992 \\
\hline
\end{tabular}

\title{
Der prähistorische Bergsturz von Pfannes/Südtirol (Italien)
}

\author{
Gerd Hering, Gunter Langheinrich \& Norbert Wilczewski *)
}

Prehistoric rockslide, petrographic research, genesis

South Tyrol, Italy

\begin{abstract}
Kurzfassung: Im Rahmen einer geologischen Diplomarbeit der Universität Göttingen wurde 1986 eine Kartierung des Bergsturzes von Pfannes im Pfitschtal/Südtirol (Provinz Bozen, Italien) durchgeführt. Die Bergsturzmassen wurden dabei petrographisch untersucht. Die Ursache des prähistorischen Bergsturzes wird in Übereinstimmung mit KLEBELSBERG (1953) darin gesehen, daß nach dem Abschmelzen des jüngsten quartären Talgletschers das Widerlager für die unterschnittene Talseite („Prallhang”) verlorenging. Die Ablösung der Bergsturzmassen erfolgte an zahlreichen tektonisch bestimmten Grenzflächen und nicht einheitlich an einer großen Bruchfläche.
\end{abstract}

[The prehistoric rockslide of Pfannes/South Tyrol (Italy)]

Abstract: As a part of a diploma thesis at the University of Göttingen the rockslide of Pfannes (Pfitschtal/South-Tyrol/ Province of Bozen/Italy) was mapped in detail in 1986.

The rockslide masses were described petrographically. In agreement with KLEBELSBERG (1953) it is concluded that the prehistoric rockslide has been caused by the melting of the youngest quaternary glacier and the subsequent loss of support for the undercut outer bank. The rockslide masses detached along numerous minor tectonic surfaces rather than along a single major fracture surface.

\section{Einleitung}

Der Bergsturz von Pfannes, auch Riegel von Pfannes oder Bergsturz in der Wöhr genannt, ist ein ausgedehntes Bergsturzareal $7 \mathrm{~km}$ nordöstlich von Sterzing (Abb. 1) in Südtirol/Provinz Bozen (Norditalien). Die Bergsturzmassen riegeln das obere gegen das untere Pfitschtal ab.

*) Adresses of the authors: Dipl. Geol. G. Hering, Prof. Dr. G. LANGheinrich, Dr. N. WilczewSKI, Institut für Geologie und Dynamik der Lithosphäre der Universität Göttingen, Goldschmidtstraße 3, 3400 Göttingen.
Im Rahmen tektonisch-petrographischer Untersuchungen in den Gesteinen der Oberen Schieferhülle am Tauernwestende (HERING 1988) wurde eine geologische Kartierung erstellt und das Bergsturzareal mit einbezogen. Auf der Basis der Kartierung wurde ein Blockblild (Abb. 2) gezeichnet, um einen besseren Eindruck vom Bergsturzareal, dem Abbruchgebiet und dem südlichen oberen Pfitschtal (Blickrichtung Norden) zu vermitteln.

Aufzeichnungen in der Kematener Kirchenchronik, Hinweise aus der Bevölkerung und Literaturrecherchen ergaben, daß unterschiedliche Vorstellungen über die Genese des Bergsturzes existieren. So sollen hier die Verhältnisse unter kritischer Würdigung der vorhandenen Literatur dargestellt werden.

Erste Erwähnung findet der Bergsturz bei KLIPSTEIN (1842). Aus morphologischer Sicht befassen sich SUPAN (1877) und DAMIAN (1894) mit dieser „Dammstufe”. LöwL (1882) deutet den Riegel von Pfannes als Bergsturz, der von der Überseilspitze kommend das Tal absperrt, während PERETTI (1935) die Ausbruchwand am gegenüberliegenden Hang (Riedspitze) vermutet. FRECH (1903) deutet die Bergsturzbrekzie als Endmoräne. KLEBELSBERG (1953) befaßt sich mit dem Bergsturz aus geomorphologischer Sicht und hält die „Aufschüttungen im Talinneren” für Bergsturzmassen, die von der Überseilspitze stammen, und der Bergsturz wird ursächlich mit dem Rückzug des letzten Gletschers in Verbindung gebracht. STAINDL (1956) folgt im wesentlichen den Ausführungen KLEBELSBERGs.

ABELE (1974) bezieht den Bergsturz in seine Auflistung von Massenbewegungen in den Alpen mit ein. HANTKE (1983) erwähnt den Bergsturz in Zusammenhang mit dem Zurückweichen des Pfitscher Gletschers im Spätwürm. 


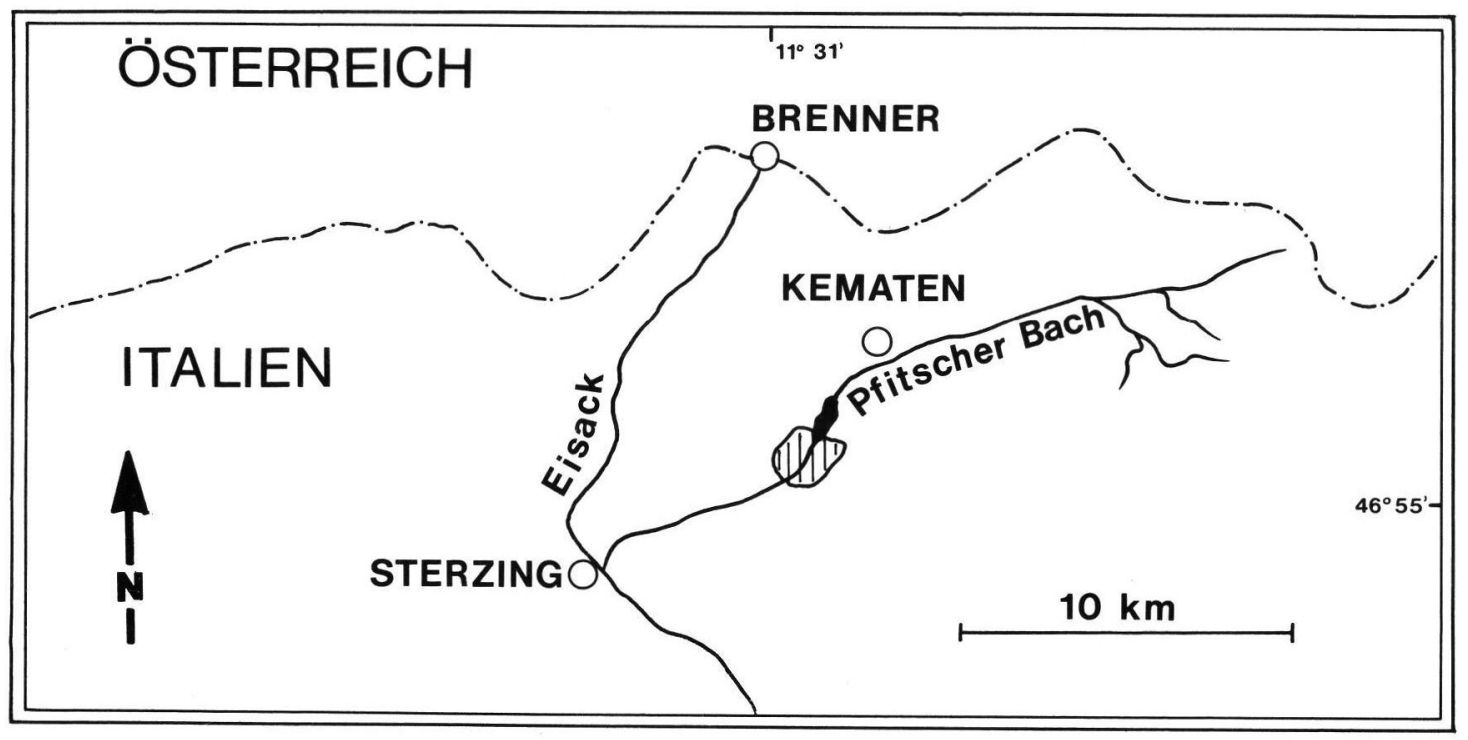

Abb. 1: Lage des Bergsturzes von Pfannes. - Bergsturzareal schraffiert.

W

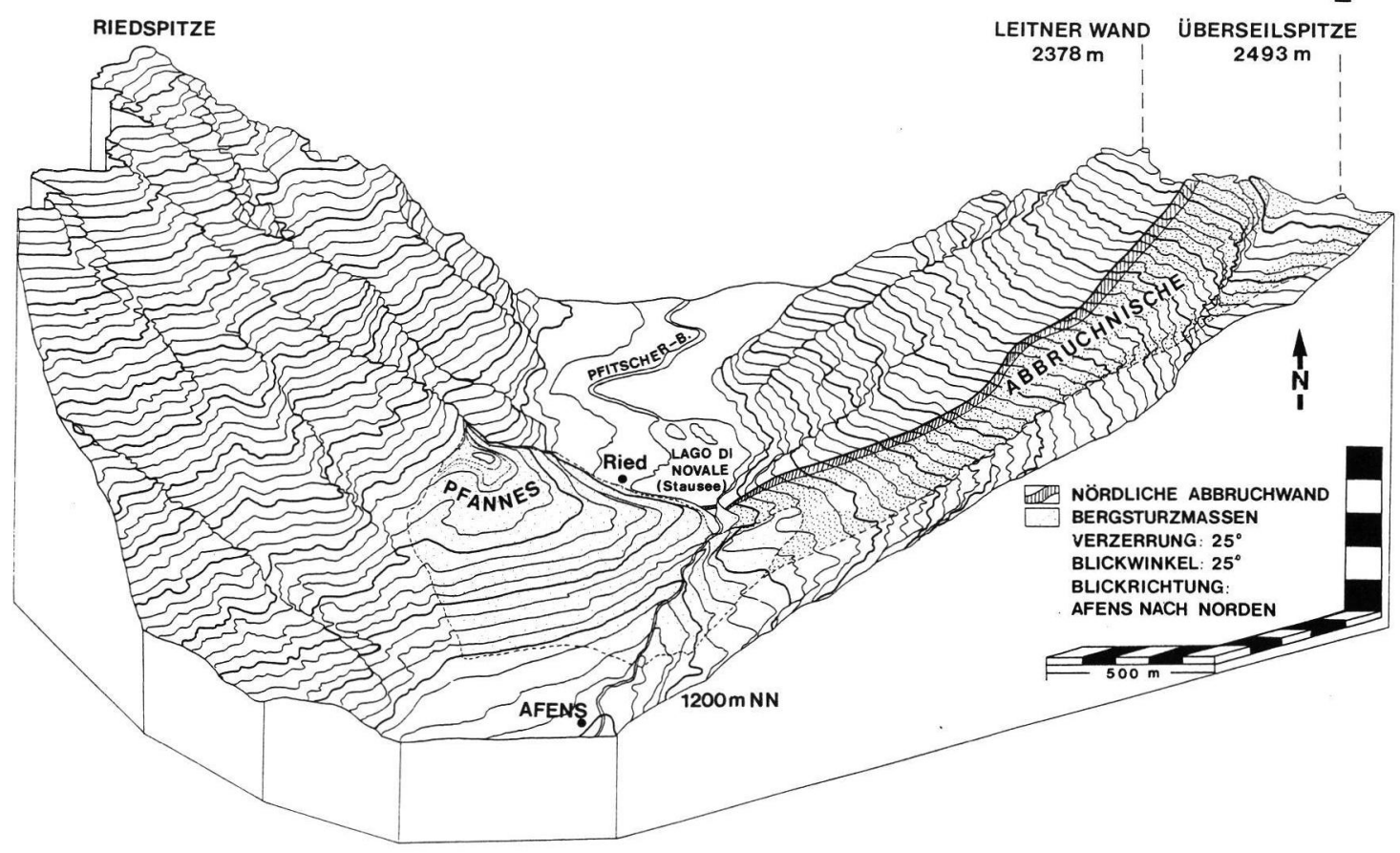

Abb. 2: Blockbild des Bergsturzareals von Pfannes mit südlichem oberen Pfitschtal. 


\section{Beschreibung}

Der Riegel von Pfannes grenzt an der westlichen Talseite auf einer Höhe von ca. $1575 \mathrm{~m} \mathrm{NN}$ an die Westwand der Riedspitze, sowie an der östlichen Talseite in Höhe der Leitner Alm (1542 m NN) an die Westwand der Überseilspitze. Er riegelt somit das Tal auf einer Breite von über $1,2 \mathrm{~km}$ ab. Im Verlauf des Tales von Ried (Novale) am Stausee $(1376 \mathrm{~m} \mathrm{NN})$ bis hin zum Südrand von Afens $(1180 \mathrm{~m} \mathrm{NN})$ erreicht er eine Längserstreckung von max. $1,3 \mathrm{~km}$.

Der Riegel von Pfannes wird durch die Bergsturzbrekzie gebildet. Sie besteht aus nahezu ungerundeten Gesteinsbruchstücken von Schluff- bis Blockkorngröße, die von calcitischer Matrix fest verkittet sind. Die Körner sind weder eingeregelt, noch nach Größe und Form sortiert. Sie bestehen aus Carbonatglimmerschiefer, Phyllit und untergeordnet Grünschiefer. Brekzie, Blöcke und Steine im Bachbett (Brücke bei $1267 \mathrm{~m} \mathrm{~N}$ ) verdanken ihre Zurundung ausschließlich dem Pfitscher Bach (Abb. 3). Es wurden keine gekritzten oder facettierten Geschiebe gefunden; eine Deutung der Bergsturzbrekzie als Moräne scheidet somit aus. Die Bergsturzbrekzie ist auch nicht mehr glazial überprägt. Die Hauptmasse der Brekzie liegt auf der westlichen Seite des Tales. Die Grenze zwischen Brekzie und Anstehendem (Gesteine der Oberen Schieferhülle) ist im Bachbett $500 \mathrm{~m}$ südwestlich Ried (Novale) aufgeschlossen. Überall sonst grenzt die Brekzie an Hangschutt. Im Pfitscher Bach wird die maximal meßbare Mächtigkeit mit 265 m erreicht. Die Grenze zum Liegenden der Brekzie ist nicht aufgeschlossen. Die Bergsturzbrekzie bedeckt eine Fläche von rund $1 \mathrm{~km}^{2}$. Ihr Gesamtvolumen wird auf $0,13 \mathrm{~km}^{3}$ geschätzt.

Im Bergsturzareal und insbesondere talabwärts bei Afens finden sich über $300 \mathrm{~m}^{3}$ große, ortsfremde Blöcke aus Carbonatglimmerschiefer, die z.T. vom jüngsten Hangschutt eingebettet sind (Abb. 4). Im Zusammenhang mit Hausgründungen sind die meisten dieser Blöcke bereits gesprengt worden. Die im Tal angehäuften Schuttmassen entstammen den Gesteinen der Oberen Schieferhülle von der Westseite der Überseilspitze. Diese Wand zeigt eine große Ausbruchnische. Im Norden zieht sie sich von „der Wöhr" bei $1500 \mathrm{~m}$ NN über eine Länge von $1,4 \mathrm{~km}$ bis zur Leitner Wand ( $2378 \mathrm{~m} \mathrm{NN}$ ). Im Osten streicht sie fast parallel zum Tal von der Leitner Wand über die Überseilspitze (2493 m NN) bis hin zur EttbergAlm. Die durchschnittliche Breite der Ausbruchnische beträgt $700 \mathrm{~m}$. Die nördliche Abbruchwand ist im unteren Teil durchschnittlich 25, im mittleren $60 \mathrm{~m}$ und im oberen bis zu $200 \mathrm{~m}$ hoch. Senkrechte und hangparallele Abbruchflächen sind im oberen Bereich der Überseilspitze deutlich zu erkennen. Sie entsprechen den Haupttrennflächen des Gebirges. Die hangparallelen Abbrüche folgen den dominierenden s-Flächen, die ungefähr senkrechten Abbrüche im wesentlichen Scharen von Hauptkluftflächen (ac, hkl).

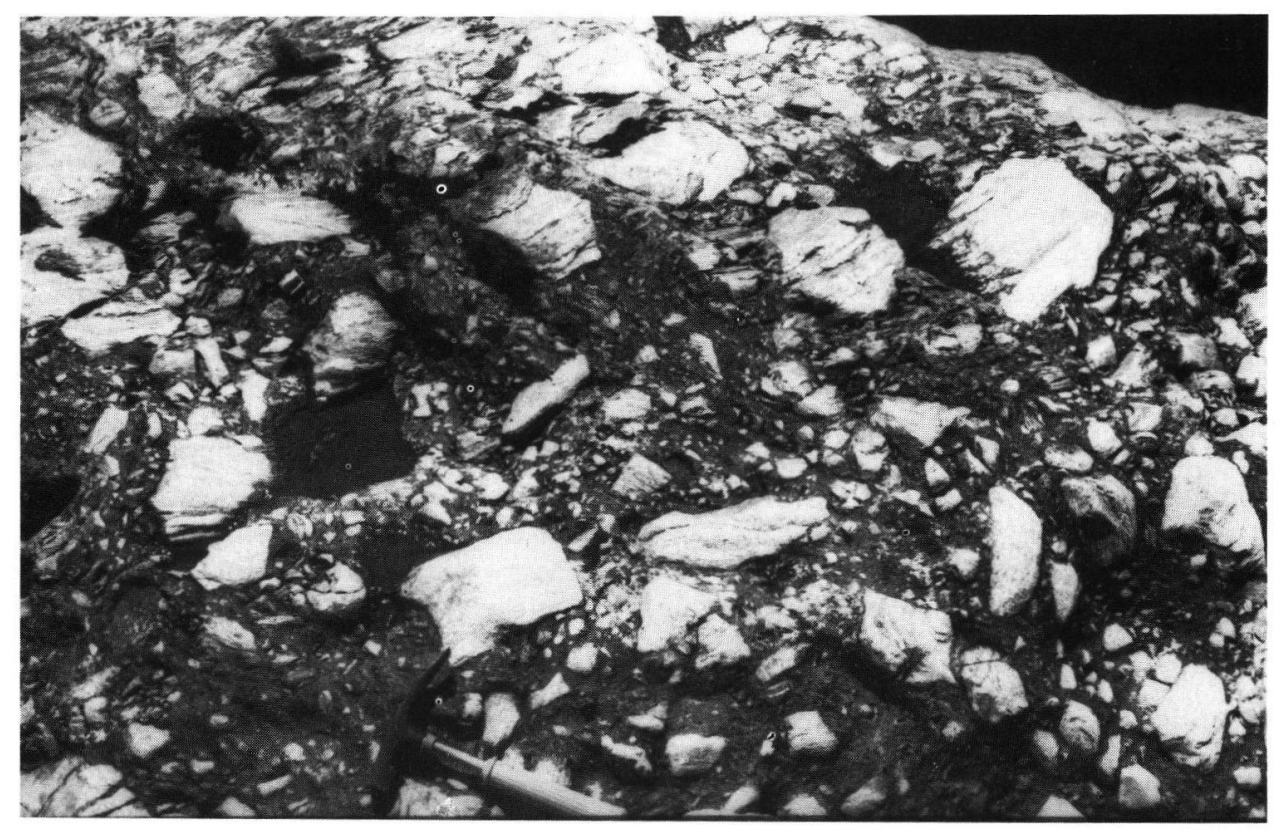

Abb. 3: Brekzie im Pfitscher Bach bei Brücke $1267 \mathrm{~m} \mathrm{NN}$. 


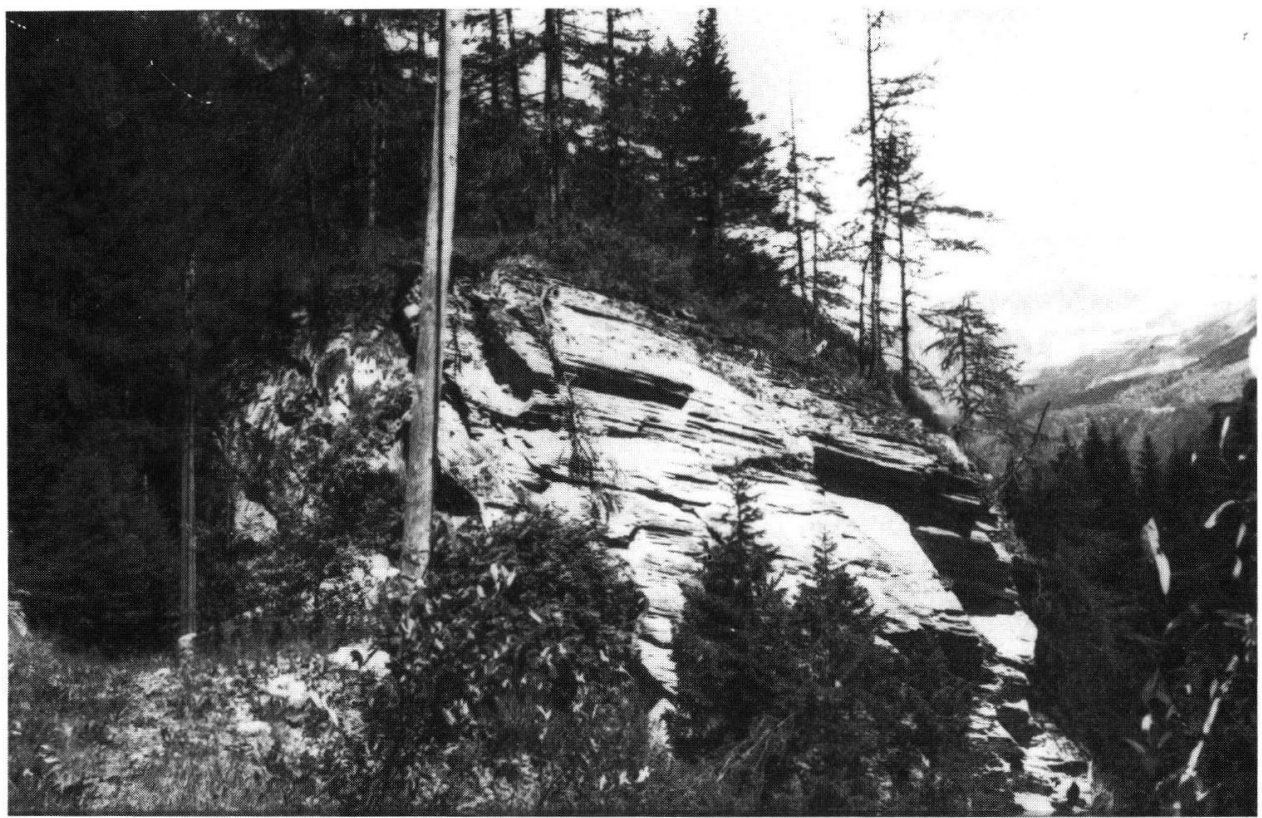

Abb. 4: Hausgroßer Block auf der Nordseite des Bergsturzes.

Die mittlere Hangneigung beträgt heute $40^{\circ}$. Der Rauminhalt der Ausbruchnische entspricht dem Volumen der herabgestürzten Felsmassen.

\section{Ursache und Bewegungsablauf}

Gletscherschrammen an den Talflanken beweisen, daß der jüngste Talgletscher (Spätwürm) die Wöhr erreichte. Dieser Gletscher hat die Gesteine der Oberen Schieferhülle am unteren Westhang der Überseilspitze über $300 \mathrm{~m}$ tief unterschnitten. In dieser Phase wurden die Felsmassen am Westhang der Überseilspitze nur noch durch den Gletscher gestützt (Abb. 5). Nach dem Rückzug des Gletschers lösten sich die Felsmassen entlang der mit $40^{\circ}$ talwärts geneigten s-Flächen.

Die der Talsohle am nächsten gelegenen Schollen wurden als erste instabil und rutschten zu Tal. Dann kamen auch die restlichen Schollen ins Gleiten und der gesamte Hang rutschte ab. Während der „Talfahrt" löste sich der Gesteinsverband auf, einzelne Schollen wurden zu Elementen von Block- bis Schluffkorngröße zerbrochen und zerrieben. Hierbei entstand Staub, der zum Aufbau der Brekzienmatrix beigetragen hat. Im Tal brandeten die Felsmassen an den gegenüberliegenden Osthang und lösten dort mehrere kleine Felsstürze aus. Einzelne Blöcke rollten und sprangen wieder zurück. Die Hauptmasse des Bergsturzes türmte sich am unteren Osthang der Ried- spitze auf und blockierte das Tal auf seiner gesamten Breite (Abb. 6). Das Hauptereignis dürfte nur wenige Minuten gedauert haben.

\section{Entstehung und Ausbruch des Pfitscher Sees}

Infolge der Talabriegelung bildete sich ein großer Stausee bis zum heutigen Dorf St. Jacob (Deckengemälde in der alten Kirche zu Kematen). Später wurde das U-Tal aufgefüllt. Die Sedimentmächtigkeit ist nicht bekannt. Der See dürfte vor dem Ausbruch eine maximale Wassertiefe von $150 \mathrm{~m}$ gehabt haben. Aus der Kirchenchronik geht hervor, daß durch Wasserableitungen alljährlich einem Dammbruch vorgebeugt wurde, weswegen der Taleingang des oberen Pfitschtales noch heute die „Wöhre” genannt wird. In der Nacht vom 18. auf den 19. März 1080 stürzte eine große Schneelawine in den Pfitscher See. Sie löste im See eine Flutwelle aus, die den natürlichen Staudamm überspülte und die Dammkrone erodierte. Die nachströmenden Wassermassen erweiterten die Bruchstelle so stark, daß der Staudamm dem Wasserdruck des Pfitscher Sees nicht mehr standhalten konnte und brach (Abb. 7). Die Flutwelle überschwemmte das untere Pfitschtal und später die Stadt Sterzing. Dieses Ereignis ist in der Kirchenchronik von Kematen/Pfitsch Seite 91 dokumentiert. Erst zu Beginn dieses Jahrhunderts wurde ein Wehr angelegt, hinter dem sich der heutige „Lago Novale” staut. 


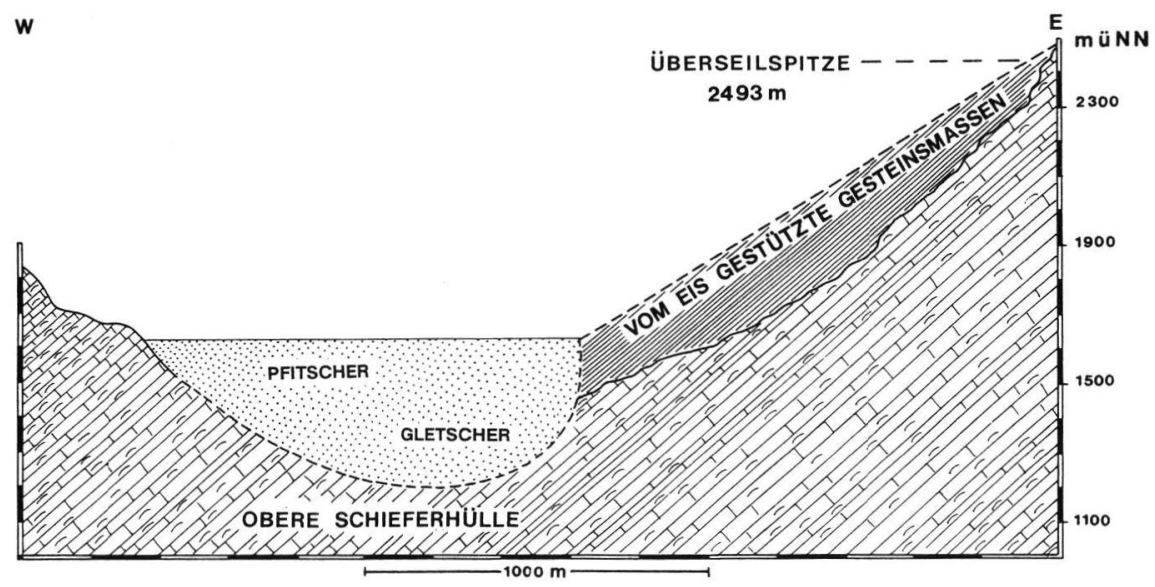

Abb. 5: Der Gletscher erodiert und unterschneidet den Westhang der Überseilspitze.

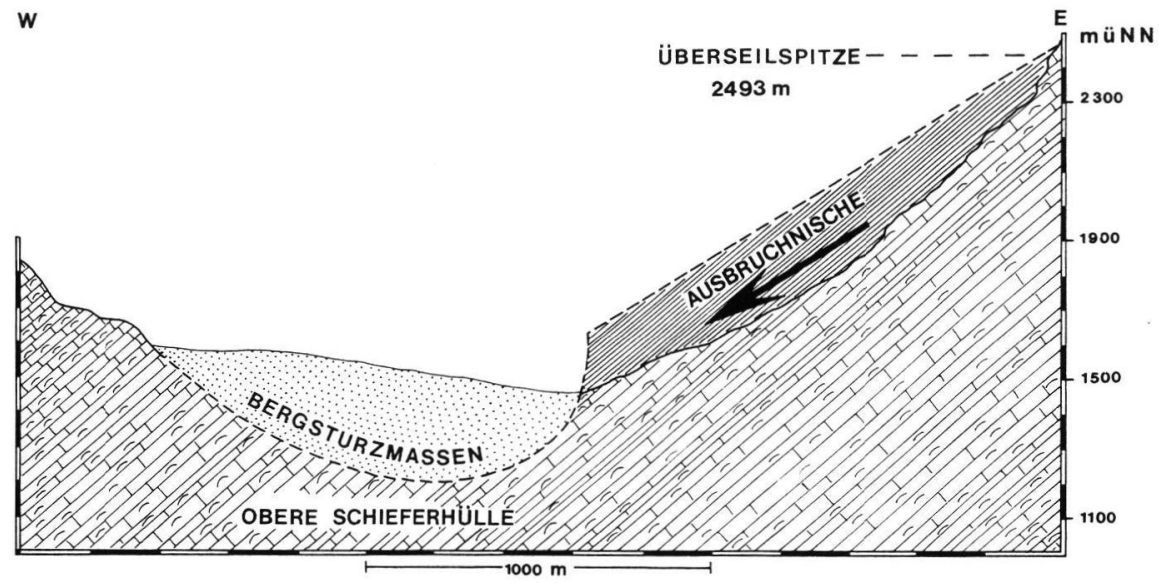

Abb. 6: Der Gletscher hat sich zurückgezogen, so daß der Westhang der Überseilspitze instabil wird. Es kommt zum Bergsturz.

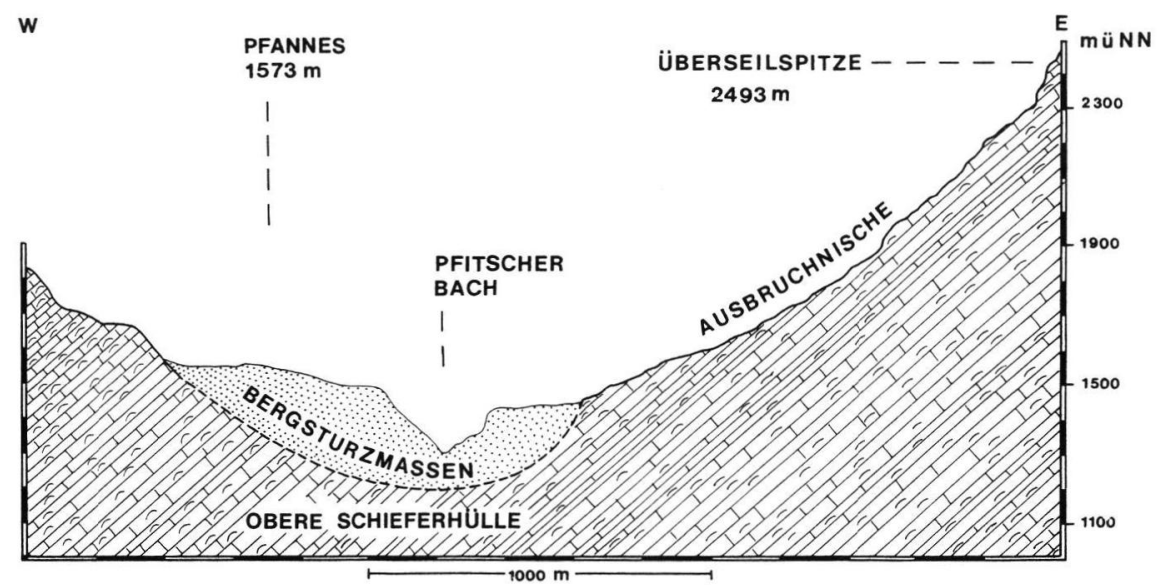

Abb. 7: Profil durch den Bergsturz von Pfannes nach dem Ausbruch des „Pfitscher Sees”.

Der Pfitscher Bach hat sich seitdem weiter durch Tiefenerosion cañonartig eingeschnitten. 


\section{Folgerungen}

Der Bergsturz von Pfannes ist eines der Beispiele in den Alpen dafür, daß durch einen Bergsturz ein natürlicher Stausee gewaltigen Ausmaßes entstehen kann. Probleme in der Beurteilung der Standfestigkeit der in diesen Fällen entstehenden Dämme stellten sich in jüngster Zeit im Veltliner Tal (VÖLK 1989). Beim Bruch der Dämme verursachen Flutwelle und Vermurung große Zerstörungen. Im vorliegenden Fall wirkte sich die von einer Schneelawine ausgelöste Flutwelle bis Sterzing aus. Dieser Bergsturz ist aber auch ein Beispiel dafür, daß natürliche Dämme mehrere tausend Jahre halten können.

\section{Schriftenverzeichnis}

Abele, G. (1974): Bergstürze in den Alpen. - Wissenschaftliche Alpenvereinshefte, H. 25, 230 S.; München.

AlverA, P. (1897): Beiträge zur Chronik von Pfitsch. unveröff. Kirchenchronik Kematen; Außerpfitsch.

DAMIAN, J. (1894): Einige wenig gewürdigte Hochgebirgsseen und erloschene Seebecken um Sterzing. - Mitt. Geogr. Ges. Wien, 37; Wien.

FreCH, F. (1903): Über das Antlitz der Tiroler Zentralalpen. Z. d. Dt. u. Österr. Alpenvereins, 34: 1-31; Innsbruck.
HANTKe, R. (1983): Eiszeit. - 3, 730 S., Ott Verlag Thun; Thun.

HERING, G. (1988): Tektonisch-petrographische Untersuchungen in der Oberen Schieferhülle am Tauernwestende. - 117 S., 47 Abb., 4 Kt., Inst. f. Geol. und Dynamik d. Lithosphäre d. Univ. Göttingen; Göttingen [Unveröff. Dipl.-Arb.].

KLEBELSBERG, R. v. (1953): Südtiroler geomorphologische Studien. Das Obere Eisacktal. - Schlernschriften, H. 113, 112 S.; Innsbruck.

Kuipstein, A. v. (1842): Geologische Fragmente aus dem Tagebuche einer Reise durch Baiern nach den östlichen Alpen. - Arch. Min., Geogn., Bergb. u. Hüttenkunde, 16; Berlin.

LÖWL, F. (1882): Über den Terrassenbau der Alptäler. Petermanns Mitt., 28: 132-143; Gotha.

Peretti, L. (1935): Morene stadiarie nelle valli delle Alpi Noriche Occidentali. - Studi Trentini di sc. nat., 16: 1-38; Trento.

StAINDL, A. (1956): Die geologischen Verhältnisse des Pfitscher Tales. - Veröff. d. Landesverbandes für Heimatpflege; Bozen.

Supan, A. G. (1877): Studien über Talbildungen. - Mitt. Geogr. Ges. Wien, 20; Wien.

VÖLK, H. (1989): Die Bergsturzkatastrophe im Veltlin 1987. Ein Modellfall für Sturzstrommobilität. - Die Geowissenschaften, 7: 1-9; Weinheim.

Manuskript eingegangen am 28. 1. 1991 